\title{
Scientific Studies on Ochradenus Aucheri Boiss
}

Mohammad Kamil*, F. Ahmad, M.T. Abdallah

ZCHRTM, DOH, A.D.UAE.

*Corresponding Author: Mohammad Kamil, F. Ahmad, ZCHRTM, DOH, A.D.UAE.

Received date: March 29, 2021; Accepted date: April 27, 2021; Published date: June 03,2021

Citation: M Kamil, F. Ahmad, M.T. Abdallah. (2021) Scientific Studies on Ochradenus Aucheri Boiss. Journal of Clinical and Laboratory Research. 2(5) DOI:10.31579/2768-0487/018

Copyright: (92021 Mohammad Kamil. This is an open-access article distributed under the terms of the Creative Commons Attribution License, which permits unrestricted use, distribution, and reproduction in any medium, provided the original author and source are credited.

Ochradenus aucheri Boiss. (Jesh) belonging to family Resedaceae usually found in sandy areas, hillsides, and mountains. Common in southern and eastern Iran to Pakistan and Muscat. Very common throughout mountains at lower elevation and on alluvial gravels in the northern Emirates. Rapidly colonizes new roadsides, e.g. around Hatta (Western, 1989). Unripe fruits eaten as treatment for digestive problems (El-Ghonemy, 1993).

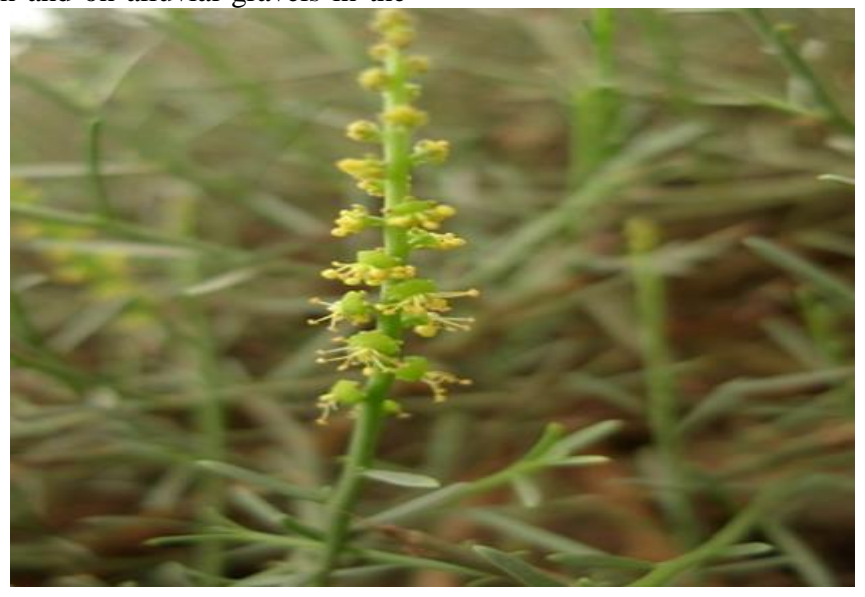

\section{Leaves and Stem}
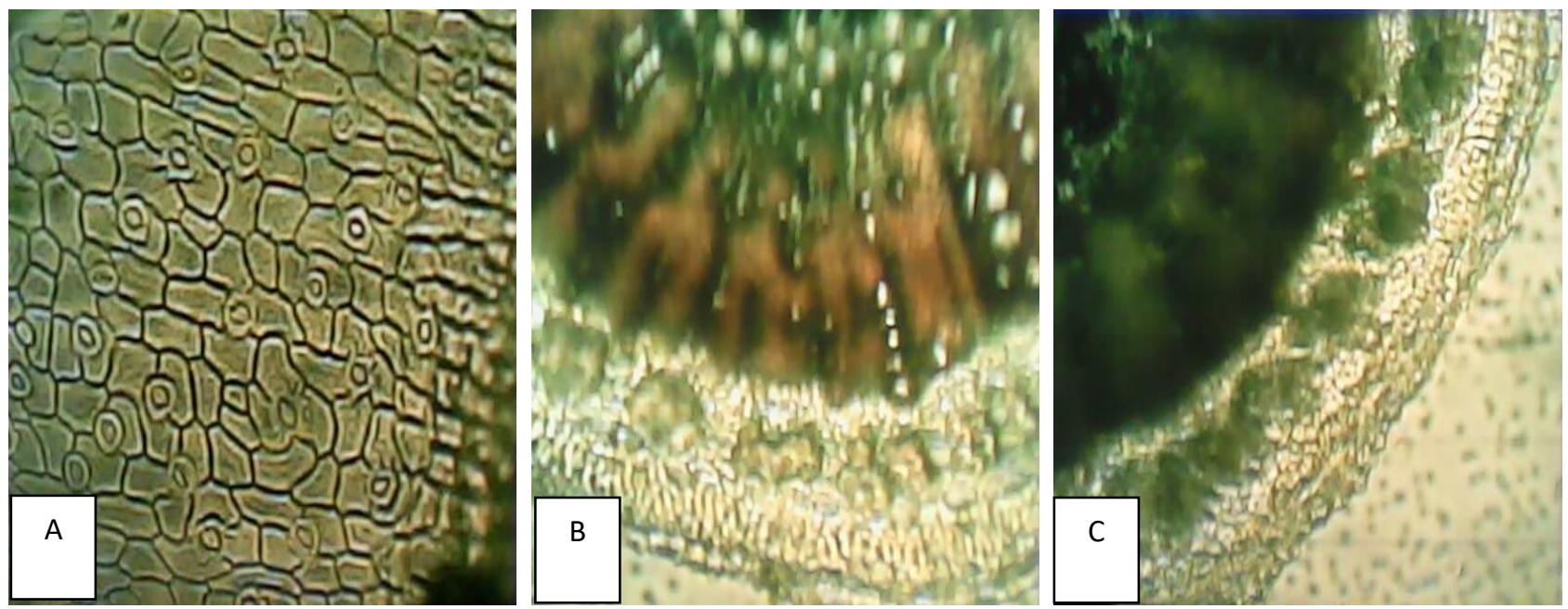

A-Surface view of the stem showing the different forms of epidermal cells with a number of oval stomata.
B-TS of a portion of the stem showing from the periphery inwards: cells of the epidermal layer, yellow cortical parenchyma cells, isolated groups of lignified fibers, layers of white distorted parenchyma cells, then 
heavily lignified vascular tissues separated longitudinally by medullary rays.

C-An oblique TS of a portion of the stem showing the patterns of the different layers constituting the stem.

\section{Chemical Constituents:}

The following chemical studies have been carried out (Quality Control Methods, 1998; Evans, 1996) on the plant Ochradenus aucheri as Phytochemical studies.

Isobornyl acetate; oleic acid; 1-Phenyl-3-butan-1-ol; palmitic acid; dimethyl diethoxy silane; stigma sterol; beta -sitosterol trimethyl silyl ester; 3',4',7-trimethyl quercitin (ZCHRTM unpublished work).

Physicochemical Constants (\%):

Loss of weight in drying at $105^{\circ} \mathrm{C} \quad: 9.20$

Absolute alcohol solubility
Water solubility

\section{Successive Extractives (\%):}

Petroleum ether $\left(60-80{ }^{\circ} \mathrm{C}\right) \quad: 0.70$

Chloroform

Absolute alcohol

Ash Values (\%):

Total ash

Water soluble ash

pH values (aqueous solution):

$\mathrm{pH}$ of $1 \%$ solution

$\mathrm{pH}$ of $10 \%$ solution

: 4.860-4.862

\section{Elemental Analyses:}

\begin{tabular}{|c|c|c|c|c|c|}
\hline \multicolumn{6}{|c|}{ Ash values ( British Herbal Pharmacopeia- Reference) } \\
\hline \multicolumn{6}{|c|}{ Assay and identification of element (AOAC International- Reference) } \\
\hline Apparatus & (AA-6800 Sh & lame method) & & & \\
\hline Element & $\begin{array}{l}\text { Std. conc. } \\
\mu \mathrm{g} / \mathrm{ml}(\mathrm{ppm})\end{array}$ & $\begin{array}{l}\text { Sample conc. } \\
\mathrm{mg} / \mathrm{ml}\end{array}$ & Sample absorbance & $\begin{array}{l}\text { Actual conc. } \\
\mathrm{mg} / \mathrm{ml}\end{array}$ & Actual conc. $(\%)$ \\
\hline $\mathrm{Cr}$ & $1,2,4$ & 20 & 0.0000 & 0.0000 & 0.0000 \\
\hline $\mathrm{Zn}$ & $0.25,0.5,1$ & 20 & 0.217 & 0.010585 & 0.0010585 \\
\hline $\mathrm{Cu}$ & $1,2,4$ & 20 & 0.0244 & 0.006895 & 0.0006895 \\
\hline $\mathrm{Fe}$ & $1,2,4$ & 20 & 0.0760 & 0.03199 & 0.003199 \\
\hline $\mathrm{K}$ & $1,2,4$ & 1 & 0.5530 & 4.976 & 0.4976 \\
\hline $\mathrm{Pb}$ & $1,2,4$ & 20 & 0.0024 & 0.00361 & 0.000361 \\
\hline $\mathrm{Cd}$ & $0.25,0.5,1$ & 20 & 0.0023 & 0.000105 & 0.0000105 \\
\hline $\mathrm{Ca}$ & $5,10,20$ & 1 & 0.0093 & 1.7447 & 0.17447 \\
\hline
\end{tabular}

$1 \mathrm{ppm}$ conc. $=1 \mu \mathrm{g} / \mathrm{ml} ;$ Actual conc. $(\%)=$ Actual conc. $(\mathrm{ppm}) \times 0.0001[1 \mathrm{ppm}=0.0001 \%]$

\section{UV Spectral Studies:}

\begin{tabular}{|c|c|c|c|c|}
\hline \multicolumn{5}{|c|}{ Ultraviolet Spectrum (USP reference) } \\
\hline Apparatus & \multicolumn{4}{|c|}{ Milton Roy Spectronic Genesys 5 Spectrophotometer - Milton Roy. } \\
\hline $\begin{array}{l}\text { Sample conc. } \\
(\mathrm{mg} / \mathrm{ml})\end{array}$ & Solvent & $\lambda \max (\mathrm{nm})$ & $\begin{array}{l}\lambda \min \\
(\mathrm{nm})\end{array}$ & Abs. $(\lambda \max -\lambda \min )$ \\
\hline 1.00 & $\begin{array}{l}\text { Intestinal Fluid simulated without pancreatic } \mathrm{pH}=7.5 \pm \\
0.1\end{array}$ & No shift & No shift & - \\
\hline 1.00 & Gastric Fluid simulated without pepsin $\mathrm{pH}=1.2 \pm 0.1$ & No shift & No shift & - \\
\hline
\end{tabular}

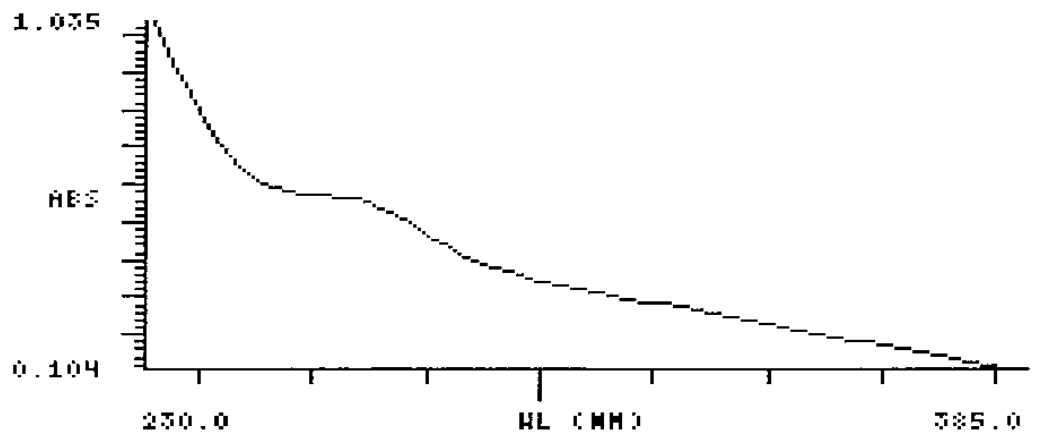

Intestinal Fluid simulated without pancreatic $\mathrm{pH}=7.5 \pm 0.1$

Thin Layer Chromatography (TLC): Wagner (1996)-Reference 

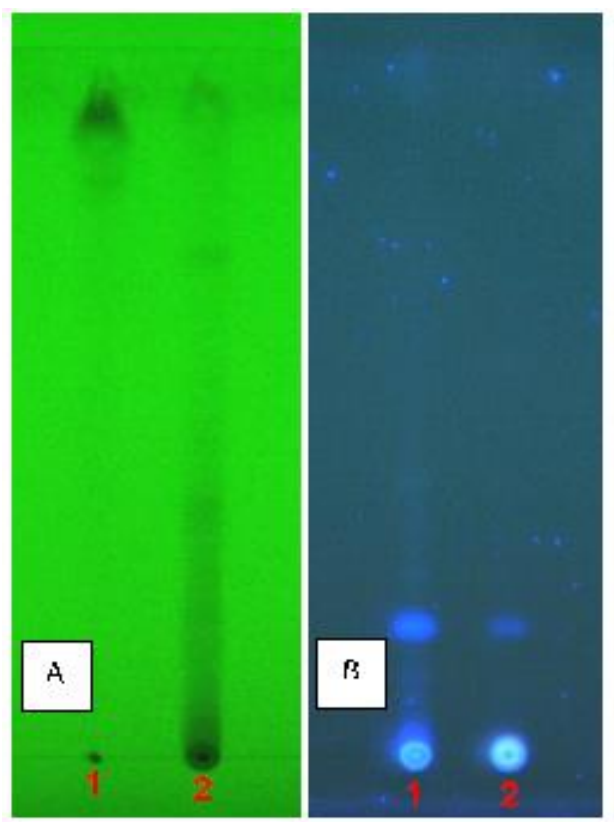

TLC fingerprint of Petroleum ether $60-80{ }^{\circ} \mathrm{C}$ (track 1 ) and $\mathrm{MeOH}$ extract (track 2)

Mobile phase 1FigA, B \& C: Ethyl acetate, methanol, water (100:13.5:10)

D: Toluene, ethyl formate, formic acid $(5: 4: 1)$

Detection
A: UV $254 \mathrm{~nm}$
B: UV366nm

Derivatization $\quad$ C \&D: Vanillin-Sulphuric acid-vis.

\section{Pharmacological and Toxicological Studies:}

Reported information literature and about the pla:nt reveals

There is not enough scientific research data and information recorded in literature about this plant; more work needs to be conducted to discover the nature and value of Ochradenus aucheri.
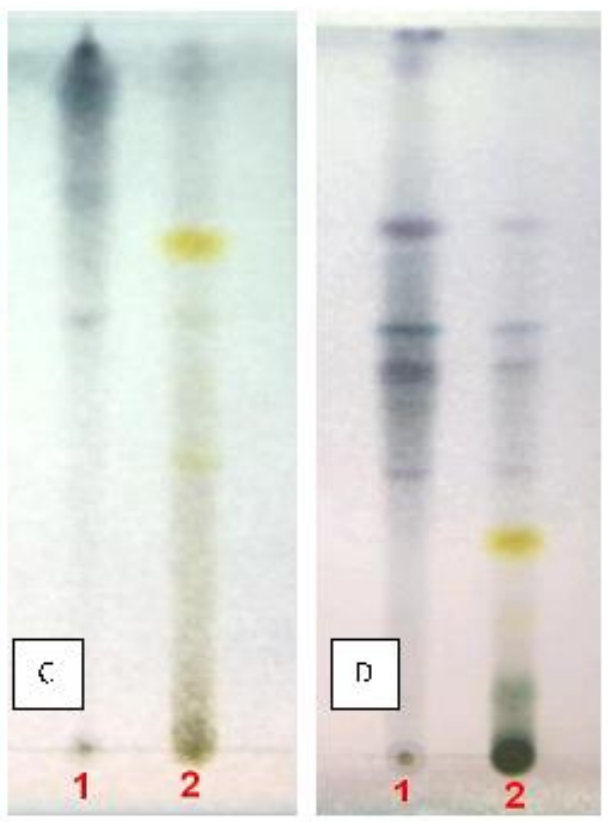

Considering the mean of accumulating heavy metals, plant species accumulating heavy metals by this order are Pergularia tomentosa, Eucalyptus sp. Convolvulus sp. Family Graminaceae, Rhiza stricta, Ochradenus baccatus. According to the mean of BAFs, heavy metal concentration of $\mathrm{Cd}$ was found to be significantly different than $\mathrm{Cu}, \mathrm{Pb}$ and $\mathrm{Zn}$. From above, these plants should be described as not-excluder and can be explored further for phytoremediation of metal polluted soils. On other hand, the practice of providing foliage and pods as fodder for livestock should be avoided (Farraj, 2007).

The following pharmacological and safety and efficacy evaluation studies were carried out on the plant aqueous extract. (Derelanko 2002; Han, 2003).

\begin{tabular}{|c|c|c|c|c|}
\hline \multirow[t]{2}{*}{ ACTIVITY } & \multicolumn{4}{|c|}{ RESULTS } \\
\hline & Strong & Moderate & Mild & Negative \\
\hline Antidiarrheal activity & $\sqrt{ }$ & & & \\
\hline Urine analysis & & & & $\sqrt{ }$ \\
\hline Effect on rabbit jejunum & $\sqrt{ }$ & & & \\
\hline Effect on rat fundus & & & $\sqrt{ }$ & \\
\hline Effect on right rat atria & & & $\sqrt{ }$ & \\
\hline Anesthetized rat (BP \& HR) & & & $\sqrt{ }$ & \\
\hline Effect on guinea pig tracheal chain & & & & $\sqrt{ }$ \\
\hline Acute toxicity & & & & $\sqrt{ }$ \\
\hline Locomotor activity test & & & & $\sqrt{ }$ \\
\hline Motor co-ordination (grip strength \& motor activity) & & & & $\sqrt{ }$ \\
\hline Rectal temperature & & & & $\sqrt{ }$ \\
\hline Body weight & & & & $\sqrt{ }$ \\
\hline Mortality & & & & $\sqrt{ }$ \\
\hline
\end{tabular}

\section{Conclusion}

The plant extract shows antidiarrheal activity and has a digestive and carminative nature. It showed no toxicity. 

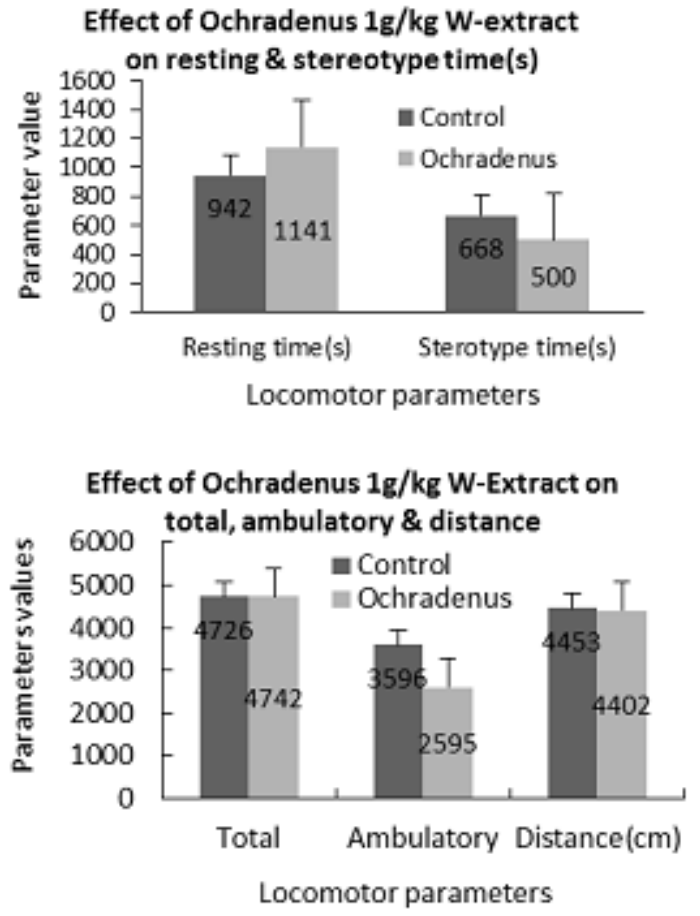

\section{Reference:}

1. Bauer A. W., Kirby W. M., Sherris J.C., \& Turck M., (1966). Antibiotic susceptibility testing by a standardized single disk method. Am J Clin Pathol; 45:493-496.

2. British Herbal Pharmacopoeia, (1996). $4^{\text {th }}$ Ed., British Herbal Medicine Association (BHMA).

3. Derelanko M. J., \& Hollinger M. A., (2002). Handbook of Toxicology. (2nd ed.). Boca Raton, USA, CRC Press.

4. El-Ghonemy A.A., (1993). Encyclopedia of Medicinal Plants of the United Arab Emirates. (1st ed.). Abu Dhabi, UAE, United Arab Emirates University.

5. Evans W.C., (1996). Trease and Evans' Pharmacognosy. (14 ed., p. 105) Saunders, London.

6. Al- Farraj A.S, \& Al-Wabel M. I., (2007). Heavy metals accumulation of some plants grown. Journal of Applied Sciences, 1170-1175.

7. Flora of Pakistan. e-floras, Web.

8. Han J., \& Hoosier G. L. V. J., (2003). Handbook of LaboratoryS, Animal Models. ( $2^{\text {nd }}$ Ed. , Vol. II). USA: CRC Press.

9. Jongbloed M. V. D., Feulner G. R., Boer B., \& Western A. R., (2003). The comprehensive guide to the wildflowers of the United Arab Emirates. Abu Dhabi, UAE, ERWDA.

10. Official Methods of Analysis of AOAC International (1999). $16^{\text {th }}$. Ed.Vol.I and II.

11. Mothana R. A. A., Abdo S. A. A., Hasson S., Althawab F. M. N., Alaghbari S. A. Z., \& Lindequist U., (2008). Antimicrobial, antioxidant and cytotoxic activities and phytochemical screening of some Yemeni medicinal plants. Evidence-Based Complementary and Alternative Medicine 7(3):323-30.

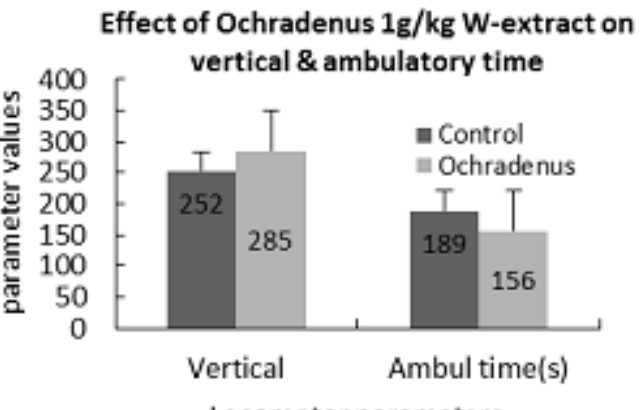

Locomotor parameters

\section{Effect of Ochradenus $1 \mathrm{~g} / \mathrm{kg}$ on rectal temperature in mice}

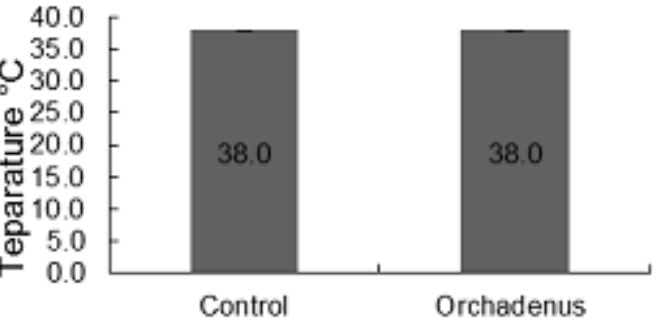

12. Quality Control Methods for Medicinal Plant Materials. (1998). World Health Organization, Geneva.

13. Wagner H., \& Bladt S., (1996). Plant Drug Analysis - A Thin Layer Chromatography Atlas. ( ${ }^{\text {nd }}$ Ed.) Springer-Verlag, Berlin Heidelberg.

14. Western A. R., (1989). The Flora of the United Arab EmiratesAn Introduction. Abu Dhabi, UAE, United Arab Emirates University.
Ready to submit your research? Choose Auctores and benefit from:
* fast, convenient online submission
* rigorous peer review by experienced research in your field
* rapid publication on acceptance
* authors retain copyrights
* unique DOI for all articles
* immediate, unrestricted online access

At Auctores, research is always in progress.

Learn more www.auctoresonline.org/journals/journal-of-clinical-andlaboratory-research 


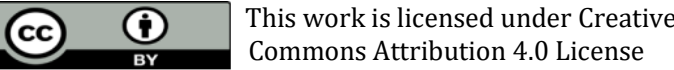

To Submit Your Article Click Here: Submit Manuscript

DOI: $10.31579 / 2768-0487 / 018$ 